\title{
Qucs Equation-Defined and Verilog-A RF device models for Harmonic Balance circuit simulation
}

\author{
Mike Brinson \\ Centre for Communications Technology \\ London Metropolitan University \\ UK \\ Email: mbrin72043@yahoo.co.uk
}

\author{
Vadim Kuznetsov \\ Bauman Moscow Technical University \\ Russia \\ Email:ra3xdh@gmail.com
}

\begin{abstract}
This paper is concerned with the development and evaluation of a number of modelling techniques which improve Qucs Harmonic Balance simulation performance of RF compact device models. Although Qucs supports conventional SPICE semiconductor device models, whose static current/voltage and dynamic charge characteristics exhibit second and higher order derivatives may not be continuous, there is no guarantee that these will function without Harmonic Balance simulation convergence problems. The same comment also applies to a number of legacy compact semiconductor device models. The modelling of semiconductor devices centered on non-linear Equation-Defined Devices and blocks of Verilog-A code, combined with linear components, is introduced. These form a class of compact macromodel that has improved Harmonic Balance simulation performance. To illustrate the presented modelling techniques RF diode and bipolar junction transistor macromodels are described and their Harmonic Balance performance simulated with Qucs and Xyce $(\mathrm{c}$.
\end{abstract}

Index Terms-Qucs, Xyce, Harmonic Balance RF simulation, compact semiconductor device modelling, equation-defined devices, macromodels.

\section{INTRODUCTION}

Since the adoption by the Qucs circuit simulation community of Equation-Defined Devices (EDD) [1] and Verilog-A analogue modules for compact device modelling [2] they have become amongst the most widely used forms of non-linear device model for established and emerging technologies [3]. The release of the open source General Public License (GPL) "Automatic Device Model Synthesizer" (ADMS) [4], has ensured that Verilog-A will remain one of the dominant compact modelling languages for the foreseeable future. Although ADMS only handles a sub-set of Verilog-A it includes a number of language statements which simplify compact semiconductor device model design [5]. Verilog-A modules and EDD models are now established as important Qucs modelling features. Qucs treats EDD models and Verilog-A modules as non-linear entities, including those with interface ports linked to internal model nodes via resistors implemented with EDD two terminal branches or Verilog-A code. This structure implies that only non-linear components are connected to internal model nodes. In general, such models function well in the DC, AC and Transient simulation domains without problems. However, the reverse is true with Harmonic Balance (HB) simulation, mainly due to problems occurring when circuits are partitioned into a frequency domain linear subcircuit and a time domain non-linear subcircuit or because of large changes in device bias points between circuit equation iterative solution steps [6]. Circuit nodes with only non-linear components connected can make partitioning difficult, often resulting in $\mathrm{HB}$ simulation non-convergence [6]. A revaluation of the role of EDD and Verilog-A modules suggests that reserving either for the nonlinear sections of an HB model reduces partition failure, provided the remaining model components are linear and at least one linear component is connected to each macromodel node. Moreover, this structure naturally builds into a compact macromodel. Non-linear EDD and Verilog-A modules with current or charge characteristics that have discontinuous differential terms can also be a source of HB simulation non-convergence. This paper introduces EDD and VerilogA macromodelling techniques which attempt to eliminate the problems found with Qucs HB circuit partitioning and device model discontinuities. Semiconductor diode and BJT Qucs HB models are described and their performance simulated with Qucs and Xyce(C)[7].

\section{MOdELling A DIODE NON-LINEAR STATIC CURRENT-VOLTAGE CHARACTERISTIC}

A basic compact device model for a semiconductor diode is shown in Fig. 1. To prevent HB floating point numerical overflow in the diode forward bias region of operation the Verilog-A function limexp is often used to calculate diode current, rather than the standard exponential function exp. When computed diode voltages have a value such that $\delta \cdot V d>80$ function limexp linearizes the diode current characteristic equation in an attempt to prevent numerical overflow. At the crossover point between the diode exponential and linear regions of operation the $I d$ and $d I d / d V d$ curves are continuous. Qucs C++ code represents real numbers using IEEE binary 64 bit real numbers. These have a decimal range of roughly $\pm 2.23 \cdot 10^{-308}$ to $\pm 1.80 \cdot 10^{308}$. Writing the diode equation given in Fig. 1 in terms of a critical voltage $V$ crit, which represents the value of $V d$ where $I d$ changes from the exponential to the linear region of operation, yields equations 1 and 2 respectively.

$$
I d=I s \cdot(\exp (\delta \cdot V d)-1), \quad \forall(V d<=V \text { crit })
$$


$I d=I s \cdot \exp (\delta \cdot V$ crit $) \cdot[1+\delta \cdot(V d-V$ crit $)], \forall(V d>V c r i t)$

where where $\exp (\delta \cdot V$ crit $)>>1.0$ and $V$ crit $=308 / \delta$ volts. For $N=1$ and $T=300$ Kelvin, $V \operatorname{crit}(\max ) \approx 7.7$ volts. Hence with $N=1$, adopting a value of $V$ crit near to, but below 7.7 volts, will ensure that values of $V d$ below $V$ crit do not cause floating point overflow when calculation Id [6]. In the exponential region of operation the first and higher order current derivatives are continuous which is ideal for $\mathrm{HB}$ simulation. However, in the linear region of operation $(V d>V c r i t)$ only the first order derivative is continuous. Figures 2 and 3 introduce a non-linear EDD model and a Verilog-A module which, when either are combined with linear resistors $R s$ and $R p$, form a compact diode macromodel. Typical simulated DC data for a diode macromodel under test are given in Fig. 4. The test circuit has $V$ crit $=0.6$ volts. This value is set artificially low in order to demonstrate the change from the exponential to the linear region of operation in the diode $I d$ characteristic. In Fig. 4 the plot of $\frac{d^{2} I d}{d V d^{2}}$ against $V d$ clearly illustrates a discontinuity at $V d=0.6$ volts.

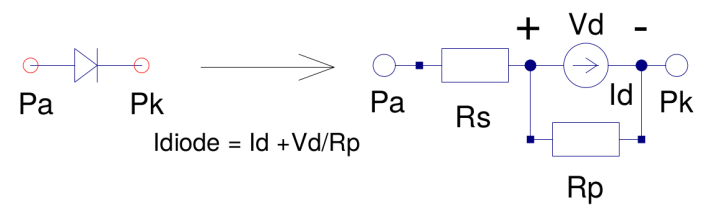

where $I d=I s \cdot(\operatorname{limexp}(\delta \cdot V d)-1), \delta=e /(N \cdot K \cdot T)$

and limexp $(X)=(X<80) ? \exp (X): \exp (80) \cdot(1-(X-80))$

Fig. 1. A semiconductor diode static $\mathrm{I} / \mathrm{V}$ model: $\mathrm{N}$ is the diode emission coefficient, e is the electron charge, $\mathrm{K}$ is the Boltzmann constant, $\mathrm{T}$ is the diode temperature in Kelvin, Rs is the series bulk and contact resistance and $\mathrm{Rp}$ a diode junction parallel leakage resistance.

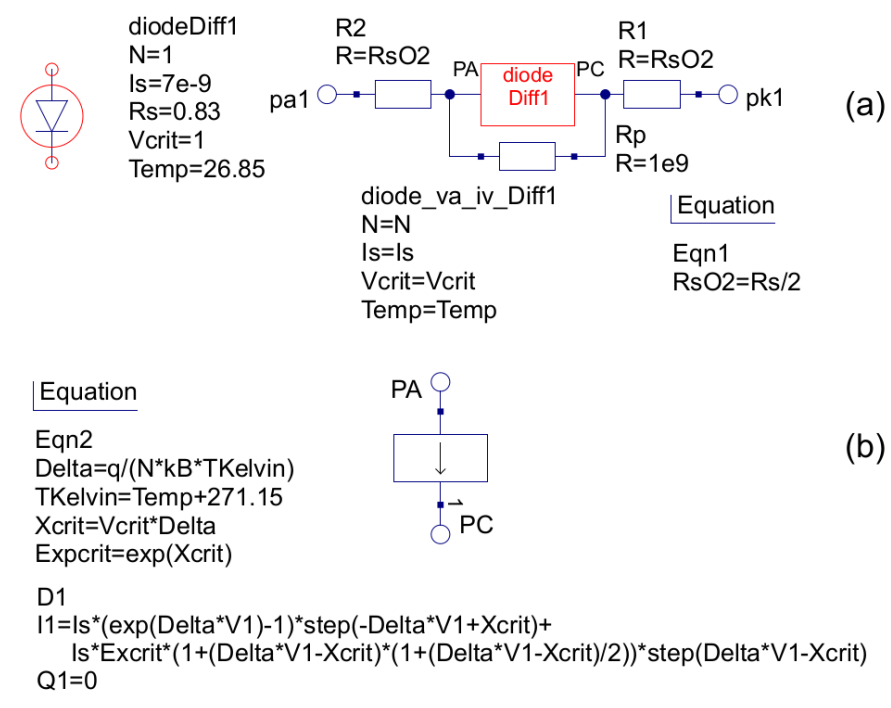

Fig. 2. A diode compact macromodel: (a) Qucs schematic symbol and macromodel circuit, (b) Qucs EDD diode current $I 1$ selected with function step () . For clarity $I 1$ is displayed on more than one line.

more general equation for the diode model current $I d$, when
$V d>V$ crit, is given by equation 3 .

$I d=I s \cdot \exp (\delta \cdot V c r i t) \cdot \sum_{i=0}^{p} \frac{\delta^{i}}{i !}(V d-V c r i t)^{i} \quad \forall(V d>V c r i t)$

where $0 \leq p \leq 5$. Illustrated in Fig. 5 are a typical set of DC simulation data obtained from the test circuit shown in Fig.4 and a diode model based on the extended macromodel defined by equation 3. In this example both Diff 1 and Diff 2 are continuous, making the model more suitable for HB simulation.

\section{MODELling DIODE NON-LINEAR DYNAMIC CHARGE CHARACTERISTICS}

Semiconductor diode diffusion and depletion capacitance are given by equations 4,5 and 6 [11].

$$
\text { Cdiff }=\frac{d Q d i f f}{d V d}=T t \cdot \frac{d I d}{d V d}
$$

where $T t$ is the diode transit time in seconds.

$$
\begin{gathered}
C d e p=\frac{d Q d e p}{d V d}=C j 0 \cdot\left[1-\frac{V d}{V j}\right]^{-M} \forall\left(V d<\frac{V j}{2}\right) \\
C d e p=2^{M} \cdot C j 0 \cdot\left[2 \cdot M \cdot \frac{V d}{V j}+(1-M)\right] \forall\left(V d>=\frac{V j}{2}\right)
\end{gathered}
$$

where $C j 0$ is the zero bias junction capacitance in Farads, $M$ is a pn junction grading coefficient, $V j$ is the junction potential voltage in Volts, and $Q \operatorname{diff}$ and $Q \operatorname{dep}$ are stored diffusion and depletion charges in Coulombs respectively. To reduce the effect of the discontinuity in equation 5 at $V d=V j$ the depletion capacitance can be represented by equations 5 and 7 .

$$
C d e p=2^{M} \cdot C j 0 \cdot \sum_{i=0}^{p} \frac{(V d-V \max )^{i}}{i !}, \forall\left(V d>=\frac{V j}{2}\right)
$$

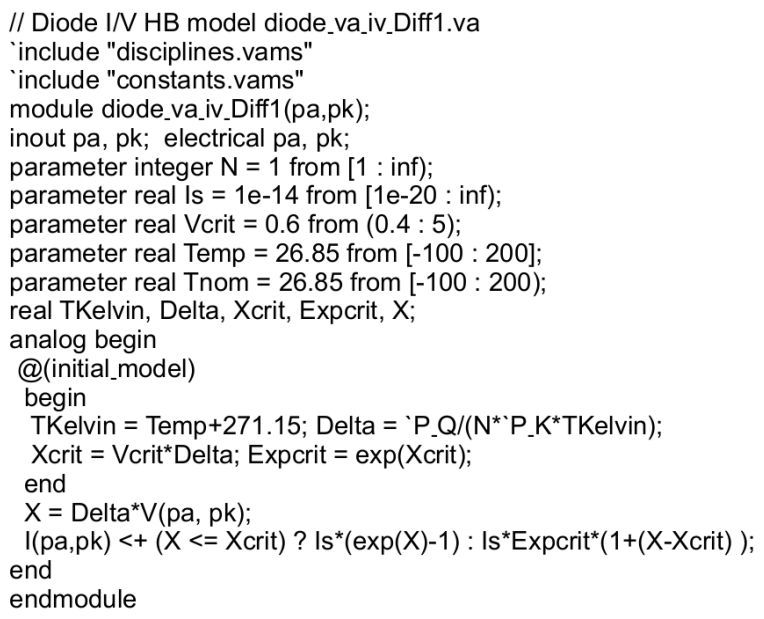

Fig. 3. Qucs diode Verilog-A code: diode current $I(p a, p k)$ selected with ternary operator $x ? y: z$. 

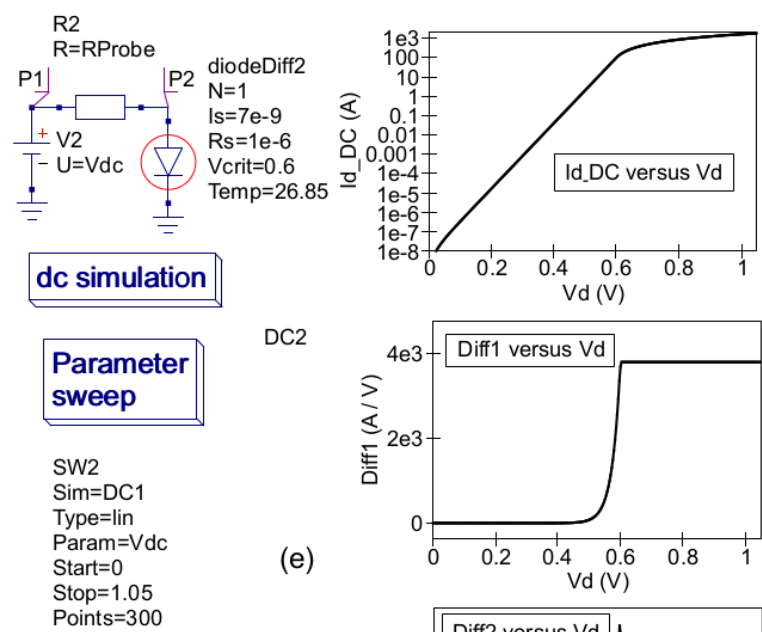

(e)

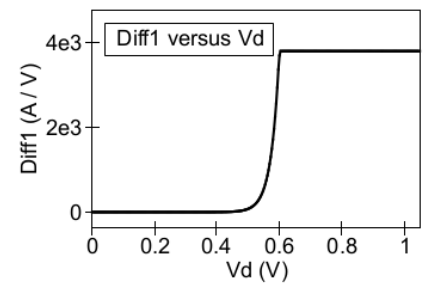

Points $=300$

Equation

Eqn2

RProbe $=1 \mathrm{e}-6$

Diff1=diff(Id DC, P2.V)

Id $\mathrm{DC}=(\mathrm{P} 1 . \mathrm{V}-\mathrm{P} 2 . \mathrm{V}) / \mathrm{RProbe}$

Diff2=diff(Diff1, P2.V)

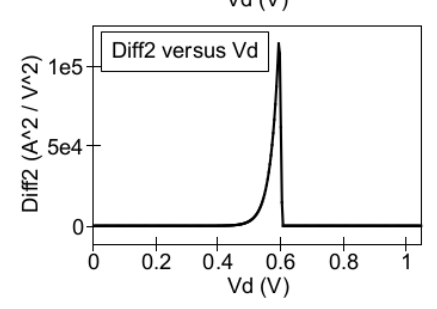

Fig. 4. Qucs compact diode macromodel test circuit and simulated DC current characteristics: $I d_{-} D C=I d, D$ iff $1=\frac{d I d}{d V d}$, and $\operatorname{Diff} 2=\frac{d^{2} I d}{d V d^{2}}$.
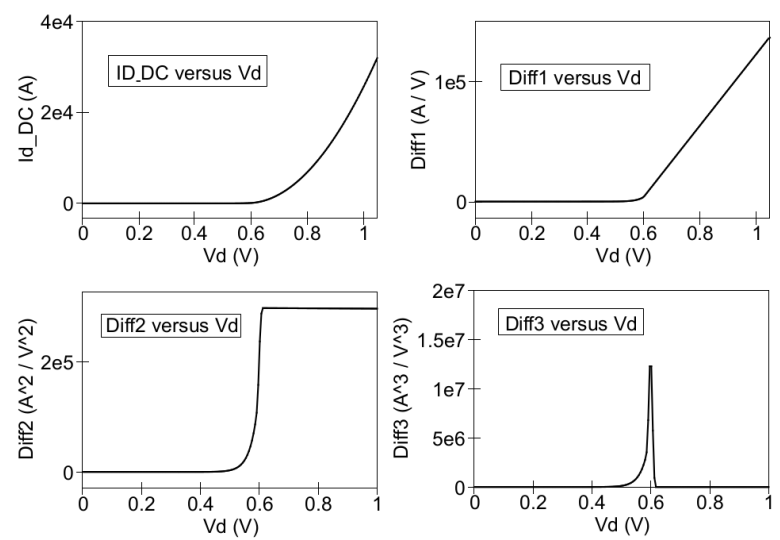

Fig. 5. Extended diode compact macromodel simulated DC current characteristics for $p=2, V$ crit $=0.6 \mathrm{~V}, I s=7 e-9 \mathrm{~A}, \mathrm{Rs}=1 e-6 \Omega$ and $T=300$ Kelvin: $I d_{-} D C=I d, D i f f 1=\frac{d I d}{d V d}, D i f f 2=\frac{d^{2} I d}{d V d^{2}}$ and Diff $3=\frac{d^{3} I d}{d V d^{3}}$.

where $0<p \leq 5$. Although equation 7 gives an approximate value for $C d e p$ at $V d>V j / 2$ it is normally acceptable because Cdiff is the dominant capacitive component in this region of diode operation. Setting $p=2$ and integrating equations 4,5 and 7 gives

$$
Q \operatorname{diff}=T t \cdot I d
$$

$$
Q d e p=C j 0 \cdot\left(\frac{V j}{(1-M)}\right) \cdot\left[1-\left(1-\frac{V d}{V j}\right)^{1-M}\right]
$$

$$
\begin{array}{r}
\forall\left(V d<\frac{V j}{2}\right) \\
\text { Qdep }=2^{M} \cdot C j 0\left[V \operatorname{diff}+\frac{V d i f f^{2}}{2}+\frac{V d i f f^{3}}{6}\right] \\
\forall\left(V d>=\frac{V j}{2}\right)
\end{array}
$$

where $V$ diff $=V d-V \max$ and $V \max =V j / 2$. A modified EDD macromodel which includes $Q \operatorname{diff}$ and $Q \operatorname{dep}$ is shown in Fig. 6. The Verilog-A code for this model is similar to the module code listed in Fig. 3 with $I(P a, P k)$ $<+\operatorname{ddt}(Q 1)$ added, where $Q 1=Q \operatorname{diff}+Q \operatorname{dep}$. Shown in Fig. 7 is a test circuit for extracting semiconductor diode capacitance from $\mathrm{S}$ parameter simulated data. In this circuit the diode under test has series resistance $R s$ set at $0.1 \mu \Omega$ to ensure that $R s$ does not affect the accuracy of the extracted values of $C d$. The diode under test has DC bias voltage $V d c$ swept over the range 0 to 0.8 volts and, at each bias point, $S[1,1]$ determined with Qucs S parameter simulation. Conversion of $S[1,1]$ to $y[1,1]$ allows diode capacitance values to be extracted from the imaginary component of the $y[1,1]$ data; $C d=\operatorname{imag}(y[1,1] /(2 \cdot \pi \cdot$ frequency $)$. Diode parameter $V j$ is set at 0.6 volts to be able to observe any discontinuities in the diode capacitance characteristics. Fig. 7 presents plots of $C d$, and its first two derivatives, with respect to diode bias voltage $V d$. These suggest that the change in depletion capacitance given by equations 5 and 7 is smooth and does not introduce any significant discontinuities in the diode capacitance characteristic.

\section{HARMONIC BALANCE AND TRANSIENT SIMULATION OF AN RF DIODE DETECTOR}

The diagram in Fig. 8 shows an unbiased RF diode detector circuit and a set of Qucs HB simulation output voltage and diode current spectral plots for a $915 \mathrm{MHz}$ five volt peak AC input signal. The detector diode model parameters are similar to the AVAGO HSMS-2820 published SPICE parameters [8].

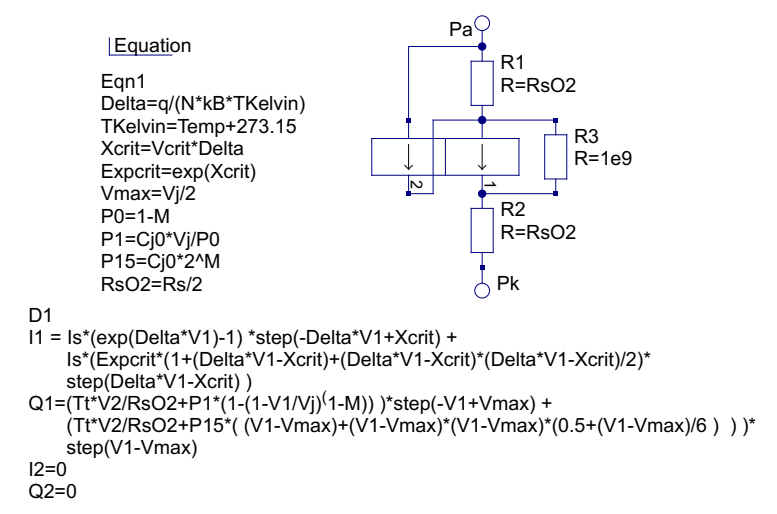

Fig. 6. A semiconductor diode EDD macromodel including dynamic charge characteristics. For clarity EDD $D 1$ current $I 1$ and charge $Q 1$ are displayed on more than one line. 

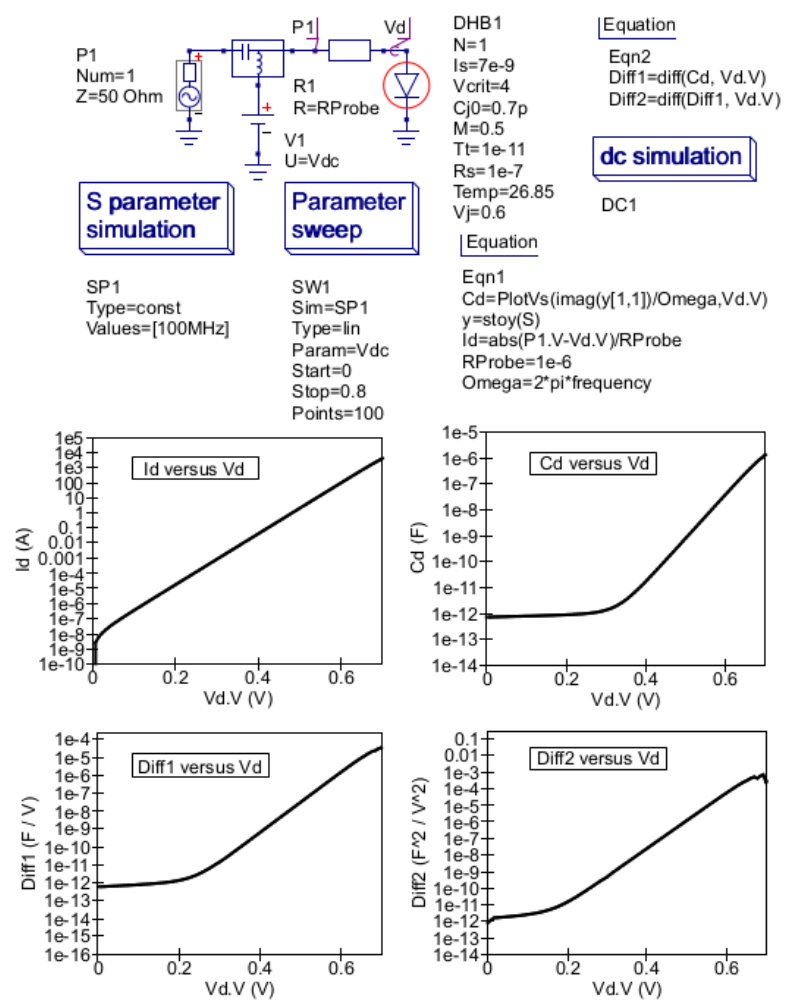

Fig. 7. Diode compact macromodel S parameter simulation: test circuit and Equation scripts for extracting diode capacitance from Qucs S parameter simulated values.

This particular circuit illustrates the performance of the diode $\mathrm{HB}$ compact macromodel and how effective $\mathrm{HB}$ simulation is in determining the AC steady state response of RF circuits, particularly when compared to number of AC input signal cycles needed before the transient simulation output voltage Nout.Vt approaches a steady state response, see Fig.9.
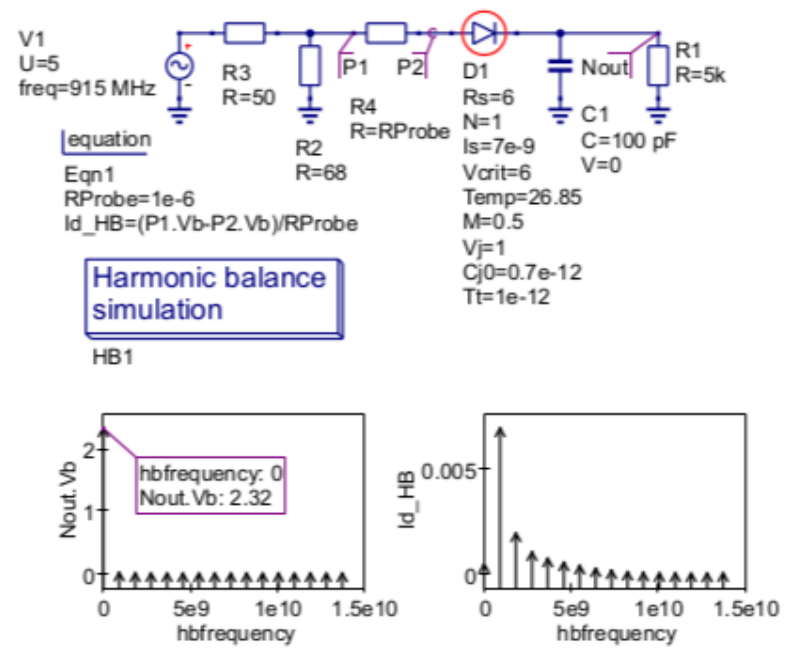

Fig. 8. RF diode detector test set and HB simulation data plots: $I s=$ $7 e-9 A, N=1, V j=1.0 \mathrm{~V}, R s=6 \Omega, C j 0=0.7 p F$ and $T t=1 e-12 s$.
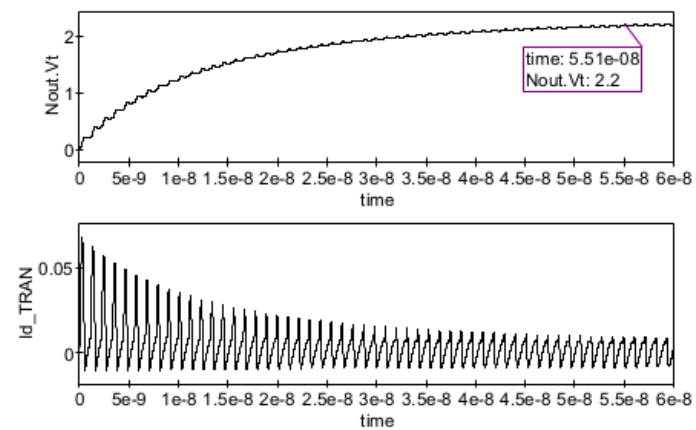

Fig. 9. RF diode detector transient simulation data plots: Nout.Vt and $I d_{-} T R A N$ against time.

\section{A BJT COMPACT MACROMODEL FOR HB SIMULATION}

A Qucs compact macromodel for an npn BJT modelled by a large signal Ebers-Moll I/V characteristic and nonlinear stored charge is given in Fig. 10. This macromodel is constructed from a nonlinear block called npnBlock and three linear resistors connecting port terminals $P C 1, P B 1$ and $P E 1$ to npnBlock. Figures 11 and 12 present details of the npn BJT nonlinear static current and dynamic charge properties derived from the semiconductor diode model introduced previously. Figure 13 introduces a basic BJT test bench and a set of Qucs HB and transient simulation derived frequency domain spectral plots for the voltage at output node $P c$. The latter being obtained with FFT techniques, see Qucs equation Eqn5 Fig. 13. DC voltage sources $V 3$ and $V$ in $D C$ were set at $15 \mathrm{~V}$ and $0.65 \mathrm{~V}$ to bias the $\mathrm{BJT}$ output node $P c$ at a quiescent DC voltage of approximately $10 \mathrm{~V}$ at a collector current of $2 \mathrm{~mA}$. Comparison of the $\mathrm{HB}$ and transient voltage spectral data for node $P c$, with $\operatorname{Vin} A C$ a single tone AC test signal of $1 \mathrm{MHz}$ frequency and $20 \mathrm{mV}$ peak amplitude, indicates good agreement between both sets of data. The latest development version of Qucs/spice4qucs [9] includes routines for generating Xyce netlists from Qucs schematics. A Xyce netlist for the npnBlock macromodel is given in Fig. 14. This netlist has a similar structure to both the Qucs EDD npnBlock model, Fig. 11, and the Verilog-A npnBlock module, Fig. 12, introduced previously. However, some minor adjustments were required

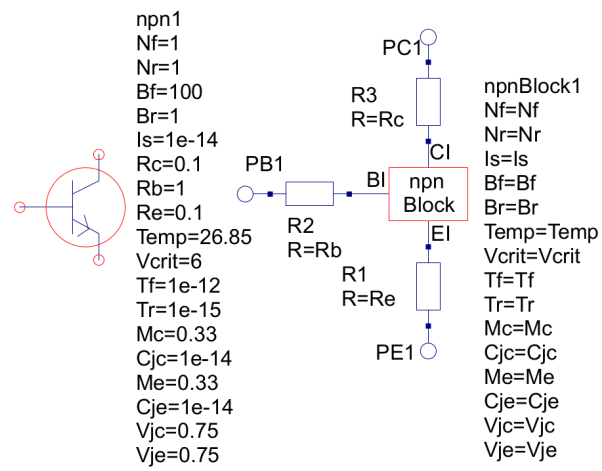

Fig. 10. An npn BJT compact macromodel: symbol and circuit. 


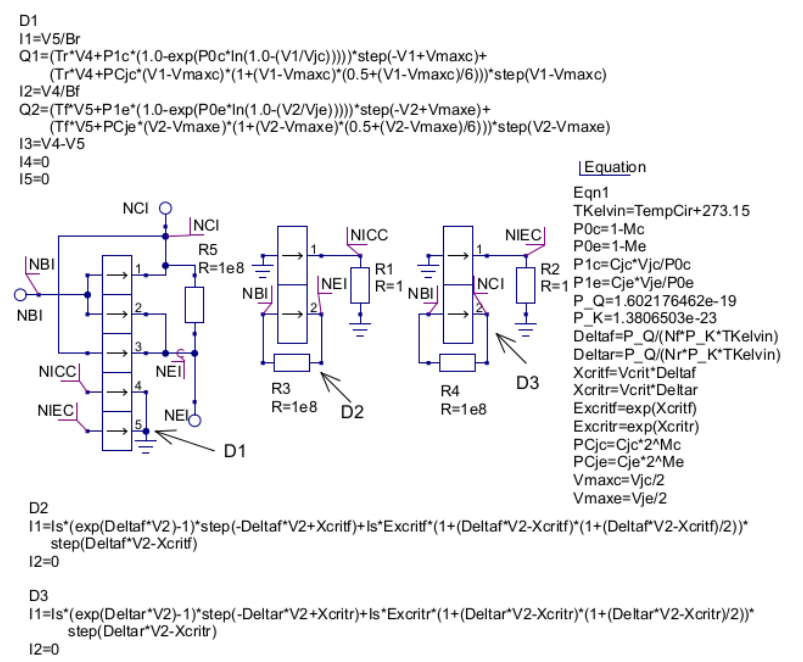

Fig. 11. A Qucs npn BJT EDD macromodel block npnBlock: For clarity EDD $D 2$ and $D 3$ currents $(I 1)$ and charge $Q 1$ are displayed on more than one line, BJT current and charge equations are selected with function step().

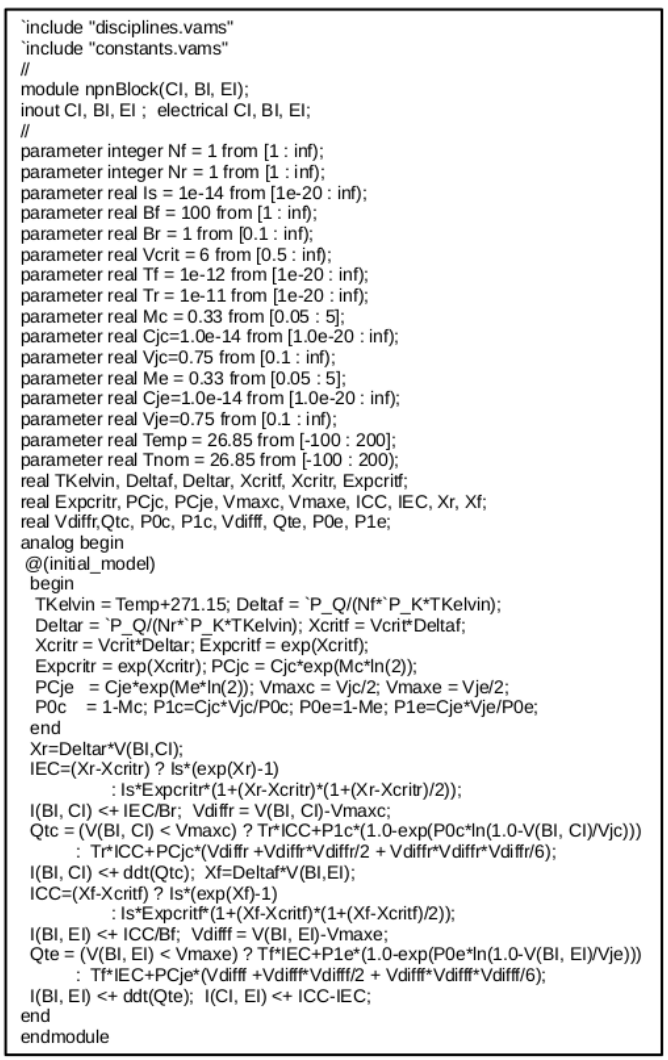

Fig. 12. A Qucs npn BJT Verilog-A code block npnBlock : BJT current and charge equations are selected with ternary operator $x ? y: z$.

to the Xyce netlist due incompatibilities in some parameter and function names, for example Qucs Temp is replaced by TempCir and Qucs function step() is replaced by Xyce function $\operatorname{stp}()$. Shown in Fig. 15 is the Xyce HB simulation spectral data for the magnitude of the voltage at test circuit node $P c$. The date illustrated in Fig. 15 confirms the values obtained with Qucs HB simulation.

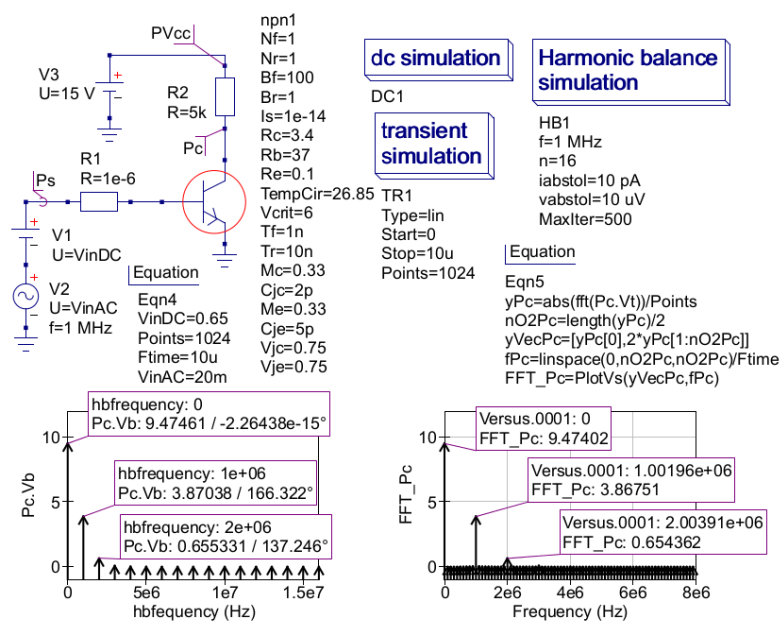

Fig. 13. A BJT HB and transient simulation test bench: Circuit, node $P C$ $\mathrm{HB}$ simulation data and frequency domain spectral plot of transient simulation data.

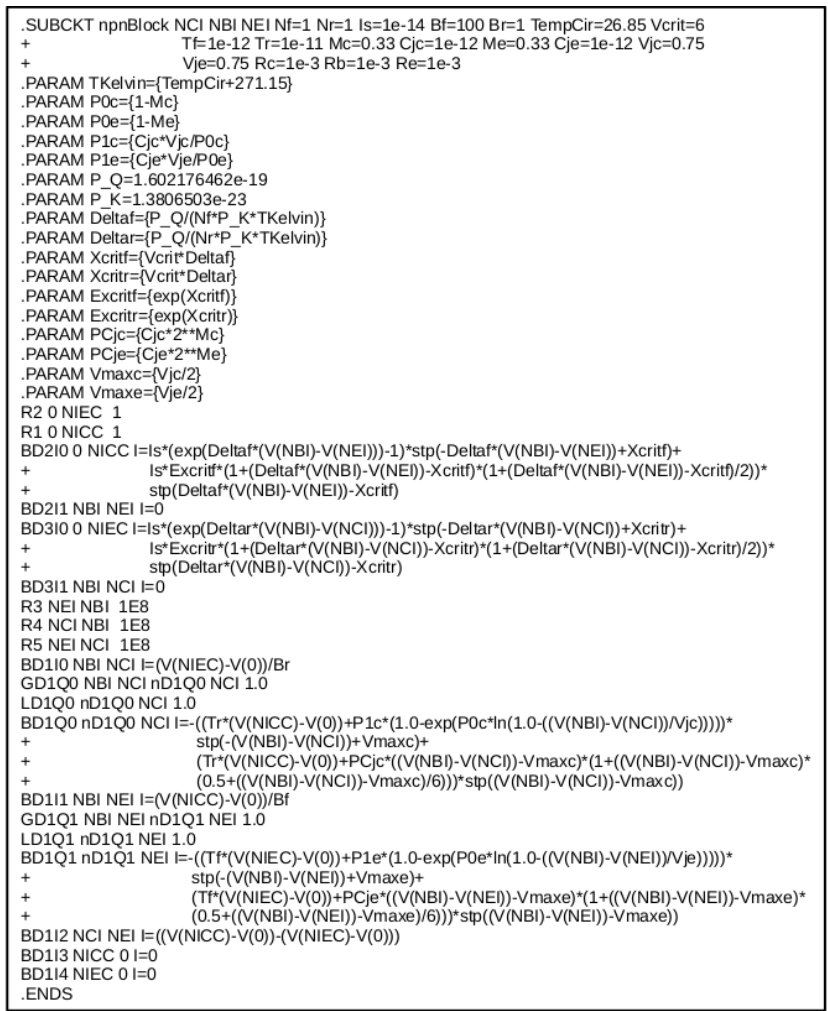

Fig. 14. A Xyce npn BJT SPICE subcircuit npnBlock: BJT current and charge equations are selected with function $\operatorname{stp}()$.

\section{AC AND HARMONIC BALANCE SIMULATION OF A SINGLE STAGE RF BJT AMPLIFIER}

The schematic for a single stage $\mathrm{Rf} B \mathrm{BJ}$ amplifier is illustrated in Fig. 16. This amplifier is designed to give a $20 \mathrm{~dB}$ voltage gain at midband frequencies. The theoretical midband 


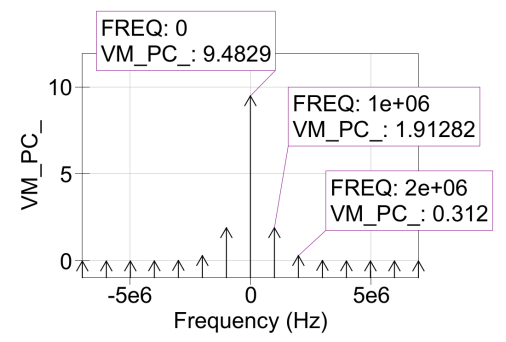

Fig. 15. Xyce HB simulation data for the magnitude of node $P c$ voltage: Test configuration identical to Fig. 13.

magnitude of the amplifier voltage gain is given by equation 11 due to the negative feedback introduced by resistor $R 9$. The Qucs plots of small signal AC and HB simulation data show very similar values for the output voltage at node Nout.

$$
\operatorname{Vgain} \approx 20 \cdot \log (R 1 / R 9)
$$

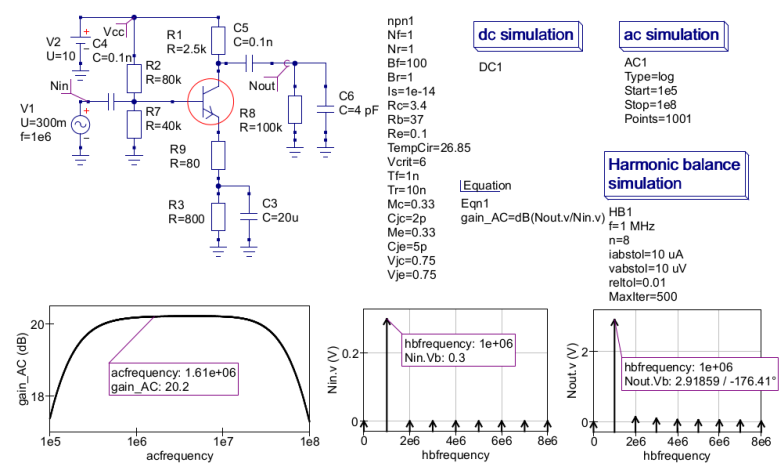

Fig. 16. A 20dB single stage npn transistor RF amplifier: circuit, small signal $\mathrm{AC}$ voltage gain and $\mathrm{HB}$ simulation data obtained with $R 9$ adjusted to give a gain of $20 \mathrm{~dB}$ at $1 \mathrm{MHz}$.

\section{CONCLUSIONS}

Harmonic Balance simulation of RF circuits is rarely implemented in GPL circuit simulators derived from Berkeley SPICE $2 \mathrm{~g} 6$ or $3 \mathrm{f5}$ [10]. Currently, Qucs includes single tone HB simulation. This paper introduces a compact macromodelling approach to Qucs HB simulation which is suitable for simulating RF discrete and integrated circuit steady state AC performance The proposed modelling technique introduces a compact macromodelling structure which reduces HB linear and non-linear circuit partitioning problems and helps reduce the effects of discontinuities in model current and charge differential characteristics on HB solution convergence. Experience with the proposed Qucs HB compact macromodelling method has shown that it is suitable for any general purpose circuit simulator provided it implements HB simulation and can handle Equation-Defined Devices or Verilog-A analogue modules. HB simulation data for a semiconductor diode and an npn BJT are reported. This data was obtained from test simulations using both the Qucs and Xyce GPL simulators.
Good agreement was found between the steady state $\mathrm{AC}$ simulation results obtained from Qucs HB simulation and transient time domain simulation.

\section{REFERENCES}

[1] S. Jahn, and M. Brinson, "Interactive Compact Modeling Using Qucs Equation-Defined Devices”, Int. J. Numer. Model. 2008, vol 21, pp. 335349.

[2] G. J. Coram, "How to (and How Not to) Write a Compact Model in Verilog-A", IEEE International Behavioural Modeling and Simulation Conference (BMAS), San José, Calfornia, pp. 97-106, October 2004.

[3] Toshi Lab. (Optical and RF-MEMS Lab), "MEMS DAIQ=MEMS Design and Analysis Interface to Qucs", 2011, http://toshi.iis.utokyo.ac.jp/toshilab/?DAIQ [accessed March 2015].

[4] L. Lemaitre, W. Grabinski and C. McAndrew, "Compact device modeling using Verilog-A and ADMS", Electron Technology Internet Journal, Vol. 35 , pp. 1-5, 2003

[5] L. Lemaitre, G. J. Coram, C. McAndrew and K. Kundert, "Extensions to Verilog-A to support compact device modeling", IEEE International Behavioral Modeling and Simulation Conference, BMAS-03, pp. 134 138. October 2003.

[6] S. A. Maas, "Nonlinear Microwave and RF Circuits", Second Edition, Artech House, Boston and London. 2003.

[7] Sandia National Laboratories, "Xyce Parallel electronic simulator: version 6.2“, 2015, https://xyce.sandia.gov/ [accessed March 2015].

[8] Avago Technologies, "HSMS-282x Surface Mount RF Schottky Barrier Diodes data sheet, Av02-1320EN, May 28, 2009.

[9] V. Kuznetsov, "QEP: Qucs schematic simulation with ngspice”, 2015, https://github.com/Qucs/qucs/wiki/QEP, (also at: https://github.com/ra3xdh/qucs/tree/spice4qucs) [accessed March 2015].

[10] A.R. Newton, D. O. Pederson, A. Sangiovanni-Vincentelli, "SPICE Version 2g User's Guide", Department of Electrical Engineering and Computer Sciences, University of California: Berkeley, CA. 1981, and B. Johnson, T. Quarles, A.R. Newton, D. O. Pederson, A. SangiovanniVincentelli, "SPICE3 Version 3f User's Manual", Department of Electrical Engineering and Computer Sciences, University of California: Berkeley, CA. 1992.

[11] P. Anongnetti, and G. Massobrio (Editors), "Semiconductor Devise Modeling with SPICE“, McGrew-Hill Inc., New York, 1988. 